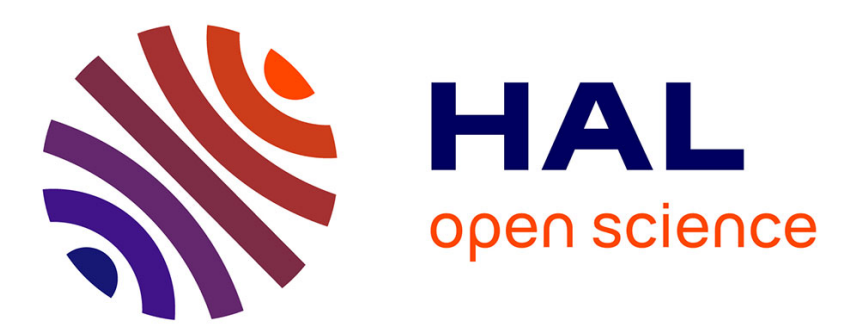

\title{
Perceiving Attitudes Expressed Through Nonverbal Behaviors in Immersive Virtual Environments
}

Brian Ravenet, Elisabetta Bevacqua, Angelo Cafaro, Magalie Ochs, Catherine Pelachaud

\section{- To cite this version:}

Brian Ravenet, Elisabetta Bevacqua, Angelo Cafaro, Magalie Ochs, Catherine Pelachaud. Perceiving Attitudes Expressed Through Nonverbal Behaviors in Immersive Virtual Environments. Motion in Games, Oct 2016, Burlingame, CA, United States. pp.175-180, 10.1145/2994258.2994280 . hal01406594

\section{HAL Id: hal-01406594 \\ https://hal.science/hal-01406594}

Submitted on 6 Dec 2016

HAL is a multi-disciplinary open access archive for the deposit and dissemination of scientific research documents, whether they are published or not. The documents may come from teaching and research institutions in France or abroad, or from public or private research centers.
L'archive ouverte pluridisciplinaire HAL, est destinée au dépôt et à la diffusion de documents scientifiques de niveau recherche, publiés ou non, émanant des établissements d'enseignement et de recherche français ou étrangers, des laboratoires publics ou privés. 


\section{Perceiving Attitudes Expressed Through Nonverbal Behaviors in Immersive Virtual Environments}

\author{
Brian Ravenet* \\ INESC-ID \\ Lisbon, Portugal \\ LTCI, CNRS, Télécom-ParisTech \\ Université Paris-Saclay \\ Paris, France \\ Magalie Ochs ${ }^{\S}$ \\ Aix Marseille Université, CNRS, ENSAM \\ Université de Toulon \\ LSIS UMR7296,13397 \\ Marseille, France
}

\author{
Elisabetta Bevacqua ${ }^{\dagger}$ \\ Lab-STICC, ENIB \\ Brest, France
}

\author{
Angelo Cafaro \\ LTCI, CNRS, Télécom-ParisTech \\ Université Paris-Saclay \\ Paris, France
}

\author{
Catherine Pelachaud \\ LTCI, CNRS, Télécom-ParisTech \\ Université Paris-Saclay \\ Paris, France
}

\begin{abstract}
Virtual Reality and immersive experiences, which allow players to share the same virtual environment as the characters of a virtual world, have gained more and more interest recently. In order to conceive these immersive virtual worlds, one of the challenges is to give to the characters that populate them the ability to express behaviors that can support the immersion. In this work, we propose a model capable of controlling and simulating a conversational group of social agents in an immersive environment. We describe this model which has been previously validated using a regular screen setting and we present a study for measuring whether users recognized the attitudes expressed by virtual agents through the realtime generated animations of nonverbal behavior in an immersive setting. Results mirrored those of the regular screen setting thus providing further insights for improving players experiences by integrating them into immersive simulated group conversations with characters that express different interpersonal attitudes.
\end{abstract}

Keywords: Virtual Agent, Nonverbal behavior, Group Simulation, Immersive System

Concepts: •Computing methodologies $\rightarrow$ Virtual reality; •Software and its engineering $\rightarrow$ Virtual worlds software;

\section{Introduction}

Interactive systems that offer an increased level of immersion have recently acquired a very high interest in both research and industrial areas, in particular in the video game industry. Two kinds of immersive systems are typically found, Head Mounted Display HMD (where the user wears a device in front of his eyes) such as the Oculus Rift ${ }^{1}$ and Spatially Immersive Display SID (where the user is surrounded by different screens) like the CAVE [Cruz-Neira et al. 1993] or the Allosphere [Amatriain et al. 2009]. Thanks to such immersive systems, the user is not just looking at a viewpoint projected on a screen, but becomes the reference of the viewpoint. By combining stereoscopic display and real-time adaptation to the user's orientation, these systems reproduce perspectives, distances

\footnotetext{
*brian.ravenet@gaips.inesc-id.pt

†bevacqua@enib.fr

$\ddagger$ cafaro@ telecom-paristech.fr

$\S$ magalie.ochs@1sis.org

๑pelachaud@ telecom-paristech.fr
}

${ }^{1}$ https://www.oculus.com/en-us/rift/ and orientations to lure the users into thinking they are within the virtual space. Previous work highlighted how these systems can lead to an increased feeling of immersion in video games [Lugrin et al. 2013] and an increased level of presence [Slater et al. 2013]. Researchers working in the domain of immersive virtual environments (IVE) usually describe presence as the feeling of actually being in the simulated environment [Cummings and Bailenson 2015; Heydarian et al. 2014]. The effect of presence or immersion on the user experience is still unclear and is still being investigated. However several works highlighted learning enhancement in educational applications [Limniou et al. 2008] with a high level of immersion; they also reported high arousal in emotional response [Diemer et al. 2015] when the feeling of presence is high. The potential of these systems could offer new opportunities for experimenting on the interactions (involving social and emotional aspects) that happen between a user and the virtual environment. More specifically, the motion of virtual characters aimed at expressing socio-emotional meaningful behavior (e.g. interpersonal attitudes) becomes important for game developers wanting to design more appealing user experiences.

However, investigating and developing applications or games with these systems raises also new research questions. It seems reasonable to wonder if the paradigms used for traditional regular 2D displays are still relevant when applied for an IVE. Camera movements, perspectives or viewpoints need to be rethought, which might lead to a different perception of the virtual world.

In this paper, we are transposing a multi-agent group simulation model, originally developed for regular computer displays and validated through an online study [Ravenet et al. 2015], into an immersive system. A particularity of this model is that it allows virtual agents to vary their animated behaviors (turn-taking strategies and nonverbal behaviors) depending on the attitudes that they aim at expressing towards each other. Attitudes can be described, following Scherer's definition of interpersonal stance [Scherer 2005], as "an affective style used naturally or strategically in an interaction with a person or a group of persons". A two-dimensional representation of interpersonal attitudes is described in [Argyle 1988]. These two dimensions are status and affiliation. In our work we adopted this representation and we define 4 possible attitudes corresponding to the two extremes of each dimension: Dominant or Submissive, Friendly or Hostile.

As opposed to the online study described in [Ravenet et al. 2015], in this study participants are immersed in the scene using a CAVE system and the simulation is running in real-time (instead of prerecorded video uploaded in the online study). The conversing 
groups that this system simulates can be used to populate a virtual world with characters expressing different interpersonal attitudes through different animated behaviors: turn-taking (using a fictitious language), accompanying gestures, rotations of the gaze and the body, and walking rearrangements into F-formations (geometrical structure of gatherings as described by Kendon [Kendon 1990]). Each of these behaviors is influenced by the attitudes an agent expresses towards the other. In this paper we describe a deployment of our model into an IVE, and a study conducted to assess whether the attitudes expressed by the virtual agents are recognized by users in this new configuration.

\section{State of the art}

In [Fox et al. 2009], the authors present a thorough review of previous research that exploits virtual reality and $3 \mathrm{D}$ environments for advancing in the field of social sciences. In particular, they highlight that in several cases users are able to recognize and produce nonverbal behaviors in a virtual environment similarly as in real world interaction. More specifically, those behaviors were interpersonal distances and gaze [Bailenson et al. 2001]. Furthermore, they show that IVEs are useful for studying social phenomena (e.g. by replicating dangerous situations in a safe environment for therapy or training) and they encourage researchers to take advantages of these technologies to perform further studies. For instance, in [Philipp et al. 2012], the authors report that the sole presence (without interaction) of virtual agents in an immersive virtual scene elicited more smile responses from participants to positive stimuli. In [Bombari et al. 2015], the authors describe how powerful it is to include virtual humans in social experiments conducted within IVE as they offer a way to standardized the interaction partners and simulate a broad range of different interactions at a very low-cost.

Other works on virtual agents and IVEs typically explored the benefit of an immersive setting over a traditional one-screen setting, in particular in learning or medical applications [Hartanto et al. 2015]. In [Patel et al. 2006], the authors presented an experiment for measuring the difference between learning tai chi through videos or an immersive virtual setting. They report that participants performed better using the IVE.

These works highlighted the potential of IVEs for simulating social settings, however, a few works addressed the issue of transferring the acquired knowledge from a one-screen display setting to an immersive one. In [McMahan et al. 2012], the authors investigated the effect of interaction and display fidelity on the user's performance, sense of presence, engagement and perceived usability of a FPS game in a CAVE setting. Their results suggest that the fidelity levels increased the user experience.

Building on our previous work and the insights provided in this section, we propose to investigate how users perceive the attitudes of a group of virtual characters simulating a conversation in an Immersive Virtual Environment. We conducted a study that follows an experimental design proposed in a previous work addressing the expression of attitudes in a traditional one-screen display setting [Ravenet et al. 2015]. However, in this paper, we deploy a behavior model into an IVE and we investigate whether this alternative configuration allows for a proper perception of the simulated agents' motions for expressing their attitudes.

\section{The Immersive Social Virtual Agents Group Simulator}

In this section we describe the system that allows us to simulate a group conversation among virtual characters that express different social attitudes towards each other through real time generated nonverbal behaviors (e.g. turn-taking behaviors, gestures or interpersonal distance). We first briefly describe the computational model of our system and then we describe its deployment in an immersive setting.

\subsection{The Social Virtual Agents Group model}

This model allows each virtual agent in a group to express an attitude towards another member, thus resulting in different behaviors being produced in real-time. An agent will be able to adapt dynamically its turn-taking behaviors, its gestures and its distances and orientations towards the other members of the group. The agents' utterances (the content of the speech) are semantically meaningless because our focus was to model nonverbal behavior while still making it possible for the agents to simulate a conversation without the influence of their verbal behavior on expressed attitudes. Therefore, we are using a fictitious language. In this way, different agents' turn-taking behaviors and utterances of different duration can be still produced, but a user cannot intervene in the conversation because the spoken language is incomprehensible. However, the agents consider the user as a (listener) member of the group and they spatially arrange in order to make space for him/her. The model is composed of three components (see figure 1), each component being responsible for a particular set of behaviors and we briefly present them here (more details can be found in [Ravenet et al. 2015] ).

\subsubsection{Turn-taking component.}

The turn-taking component establishes when an agent starts or stops talking, when it interrupts the others or when it is interrupted itself depending on which agents are currently speaking and on the attitudes it expresses towards each other. This component works as a state machine (inspired by [Thórisson et al. 2010]) and it alternates the agent's role between speaker and listener (according to Goffman's definition of conversational roles [Goffman 1981]). The states and transitions were designed following the literature on turntaking, conversational agents and attitude expression.

\subsubsection{Group Behavior component.}

The Group Behavior component changes the desired target for the agent's gaze and body orientation. It also computes the desired interpersonal distances for an agent towards all other members of the group (including the user). The final computation of the gaze, orientation and distance is supported by an adapted version of the Impulsion Social AI Engine [Pedica et al. 2010]. The rules for the adaptation of these behaviors were constructed following the literature on small group formations (c.f. F-formations [Kendon 1990]), interpersonal distances (c.f. proxemics theory [Hall 1969]) and attitude expression.

\subsubsection{Conversational Behavior Component.}

The conversational Behavior component produces nonverbal behaviors accompanying the utterances of the virtual agent and it varies the behaviors that are being produced depending on the attitudes that the agent aim to express. It can influence the amplitude and the strength of its gestures along with its facial expression (positive, negative or neutral). It takes the attitudes as input and alters the nonverbal signals produced by the Behavior Planner of the Greta/VIB system [Pecune et al. 2014] thanks to the model presented in [Ravenet et al. 2013]. This model is based on a machine learning approach. A Bayesian classifier was learned from crowdsourced data of agent behaviors expressing attitudes and a model 


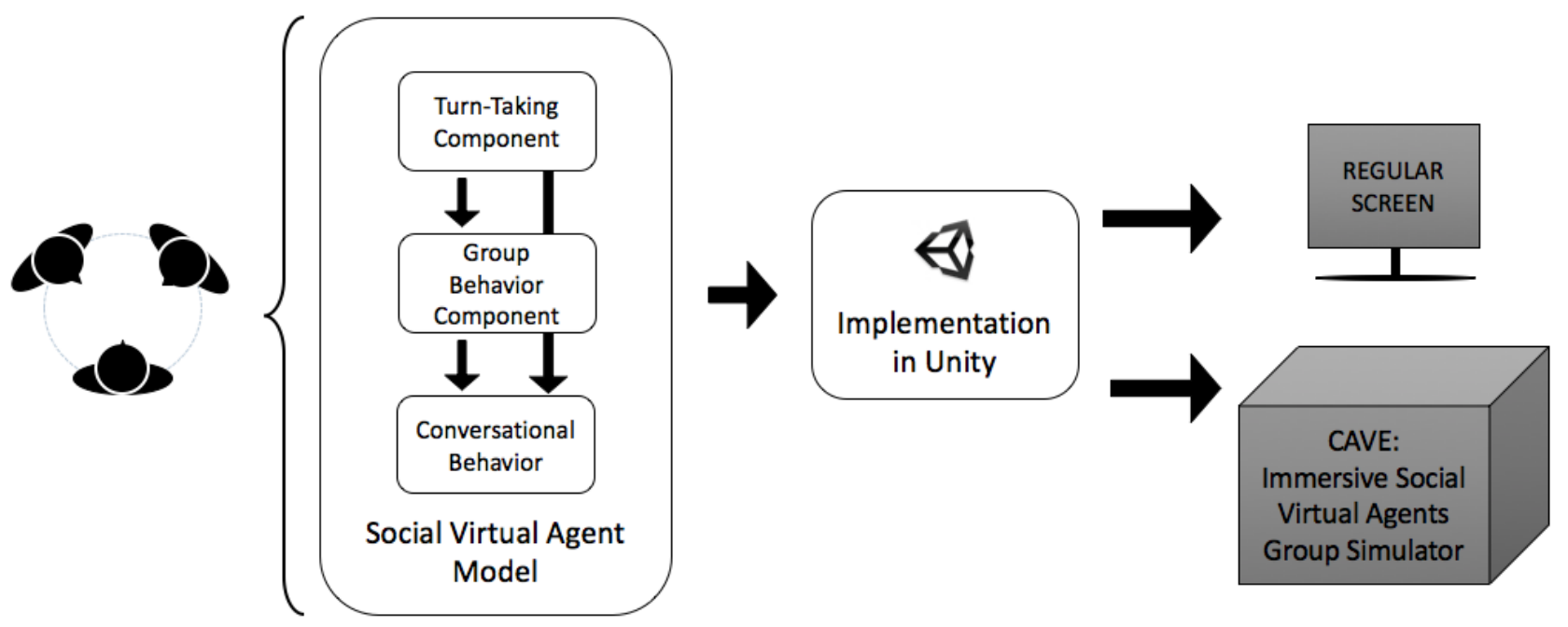

Figure 1: The architecture of the Immersive Social Virtual Agents Group Simulator.

for expressing these attitudes was built upon it.

The full model has been implemented in Unity3D, similarly to the online study conducted in [Ravenet et al. 2015]. Benefiting from the portability of the Unity3D framework, we deployed the system in an immersive CAVE system at the CERV laboratory in Brest. In the next section, we give more details on the technical aspects of this implementation.

\subsection{The CAVE system}

The CAVE system displays a virtual environment on four screens, using stereoscopic images, that surround the user [Cruz-Neira et al. 1993]. Three wall screens are set in front of the user, on his right and on his left and a 6 square meters-size ground screen below the user. This immersive system works with four short focal length projectors which are directed toward the three walls and the face on the ground. For tracking purposes, we used OptiTrack motion capture system and Motive software ${ }^{2}$. The OptiTrack is composed of eight infrared cameras placed on the top of the frame circumnavigating around the capture volume. The cameras can track the user's head thanks to passive sensors attached to the 3D glasses the user is wearing to visualize the stereoscopic rendered scene. The user's head position is used in Unity3D to place the camera and then to continually redrawn the virtual environment to preserve the viewer's perspective.

In this configuration, we added in our model the capacity to map the location of the glasses in the real world (thanks to the OptiTrack system) to the location of the user in the virtual space. This allows us to be able to represent the user as a physical entity in the virtual scene (i.e. another agent), in order for the virtual agents to place themselves in a circle that includes the user in their group. In the current setting, the agents are considering only the physical proximity of the user in order to include him/her in the group formation but they are not addressing him/her their turn, because of the fictitious language that they use.

The fully implemented system supports the creation of different groups of characters within a scene that simulate conversations. The virtual characters can express different attitudes towards each other (Dominant, Submissive, Friendly or Hostile). The user can

\footnotetext{
${ }^{2} \mathrm{https}: / /$ www.optitrack.com
}

typically approach a group and join a conversation and the agents react (in real-time) by (re)arranging themselves in order to make physical space and keep the F-formation system. However, in this study participants were standing still while observing the simulated group conversation as part of the group (i.e. we controlled for their motion because this would have added an additional bias to the conditions if, for example, they would look at the agents from different distances from the group members).

\section{Evaluation}

\subsection{Objectives}

The objectives of our evaluation was to assess whether the attitudes expressed by a group of agents through their behaviors, such as getting closer for expressing a friendly attitude for instance, would be correctly recognized by the participants when immersed in the virtual scene inside the group. It was unpractical to test all possible group configurations, we fixed the number of agents in our conditions to 4 ( 2 males and 2 females). We also divided the study in two trials. In the first trial (Status Trial), the agents could express only attitudes on the Status axis (Dominant vs. Submissive) and in the second trial (Affiliation Trial), the agents could express only attitudes on the Affiliation axis (Friendly vs. Hostile). To avoid any bias due to the appearance and the gender of the agents we determined 12 different group configurations to assure an equitable presentation of the four agents and the attitudes ( 6 in each trial).

Each participant was assigned to a trial and to a specific group configuration. They observed 4 conversations (each lasting one minute). Even though the participants were not involved in the verbal conversation, they were still part of the group surrounded by the agents. We asked participants to observe with particular attention the two central agents, with respect to the four agents in the group, that were in front of them. These two agents, that we named Left Agent and Right Agent, expressed differently their attitude following Sadler's intercomplementarity theory [Sadler and Woody 2010]:

i) In two conversations they expressed complementary attitudes, which are opposite on the status axis (i.e. dominant and submissive) or similar on the affiliation axis (i.e. friendly and friendly) 
ii) In the other two conversations they showed anti-complementary attitudes, which are opposite on the affiliation axis (i.e. friendly and hostile) or similar on the status axis (i.e. dominant and dominant).

Therefore, we defined the following independent variables:

- The Expressed Status of Left Agent, which corresponds to the status expressed by the Left Agent in the Status trial (similarly we define The Expressed Affiliation of Left Agent in the Affiliation trial).

- The Expressed Status of Right Agent, which corresponds to the status expressed by the Right Agent in the Status trial (similarly we define The Expressed Affiliation of Right Agent in the Affiliation trial).

Each variable had two levels, dominant and submissive in the Status trial (hostile and friendly in the Affiliation trial). We also defined the following dependent variables:

- The Measured Status of Left Agent, which corresponds to the user perception of the status of the Left Agent in the Status trial (similarly we define The Measured Affiliation of Left Agent in the Affiliation trial).

- The Measured Status of Right Agent, which corresponds to the user perception of the status of the Right Agent in the Status trial (similarly we define The Measured Affiliation of Left Agent in the Affiliation trial).

\subsection{Hypotheses}

Based on the results of the previous online study, we formulated the following hypotheses:

- H1.S: The Left Agent is perceived more dominant when it expresses a dominant attitude than when it expresses a submissive attitude.

- H1.A: The Left Agent is perceived more friendly when it expresses a friendly attitude than when it expresses a hostile attitude.

- H2.S: The Right Agent is perceived more dominant when it expresses a dominant attitude than when it expresses a submissive attitude.

- H2.A: The Right Agent is perceived more friendly when it expresses a friendly attitude than when it expresses a hostile attitude.

\subsection{Procedure}

In order to assess the participants' perception of the agents' attitudes, we used an adaptation of the questionnaire provided in [Funder et al. 2000]. We designed a questionnaire for each trial. For each measured attitude, we selected 4 items (i.e. those with higher reliability), 2 with positive valence and 2 with negative valence. The questions are summarized in Table 1. All questions used a 5points Likert scale (anchors: Completely Disagree and Completely Agree).

\subsection{Participants}

Twenty-four subjects, twelve for each trial, were recruited.

In the Status trial, $16.7 \%$ were women and $83.3 \%$ men, they were mostly from 31 to 40 years old $(58.3 \%)$.

In the Affiliation trial $25 \%$ of the subjects were women, and $75 \%$ were men, mainly aged from 21 to $30(66.66 \%)$.
Almost all the participants were French $(100 \%$ in the Status trial and $83 \%$ in the Affiliation trial), we had two foreign ones from Lebanon and Madagascar.

Each participant was assigned to a specific group configuration and then observed 4 simulated conversations (we had two participants assigned to each possible group configuration) in a fully counterbalanced order, thus avoiding first and second carryover effects [Bradley 1958].

We invited participants in the CAVE and explained to them the study's procedure while already wearing the glasses for 3D vision and the head tracking system. We showed a tutorial were all agents were expressing neutral attitudes. We used life-sized agents that, at the beginning of each condition, placed themselves in a circle in order to include the participant and then started the simulated conversation (see Figure 2).

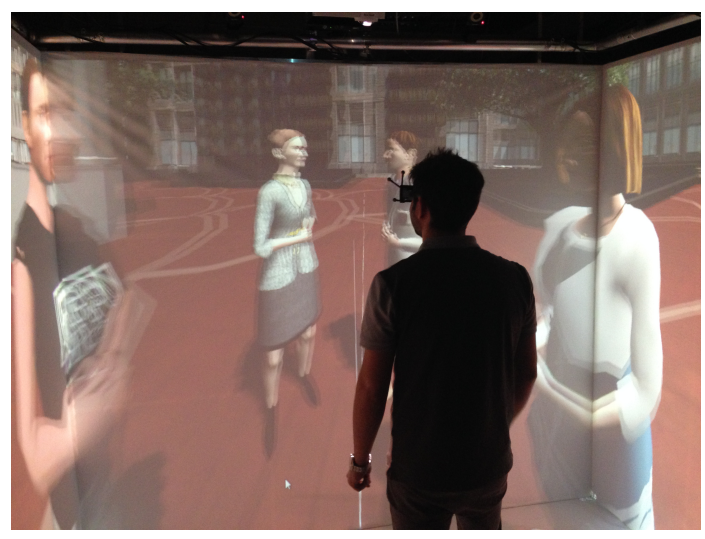

Figure 2: Experiment setting. The users must maintain their position however they are free to look around.

During the experiment, after each stimuli of about 1 minute, participants filled in on a separate desktop PC the questionnaire about their subjective evaluation of the agents' attitudes in the condition just observed.

\subsection{Results}

We combine the answers to the questions in order to obtain a single normalized value for each dependent variable.

\subsubsection{Status trial.}

We first conducted a MANOVA $2 \times 2$ on our variables. A main effect of the expressed Status of Left Agent (WilksLambda $=0.36$, $F(2,10)=8.9, p<0.05)$ and the expressed Status of Right agent (WilksLambda $=0.29, F(2,10)=12.5, p<0.005)$ were identified, which could indicate that varying the expressed attitudes has an effect on the measured attitudes. Additionally, no effect of the interaction of the independent variables was identified.

Following-up, we ran an ANOVA for each dependent variable. The expressed Status of Left Agent had a significant effect on its measured Status $(F(1,11)=18.24, p<.005)$. The measured Status of Left Agent was higher when the Left Agent was expressing Dominance $(M=.67, S E=.04)$ than Submissiveness $(M=.40$, $S E=.02)$.

A similar effect was identified for the effect of the expressed Status of Right Agent on its measured Status $(F(1,11)=15.8, p<.005)$ with a higher value for the measured Status when the expressed Status was Dominant $(M=.66, S E=.04)$ than Submissive 


\begin{tabular}{|l|l|l|l|}
\hline \multicolumn{2}{|c|}{ Affiliation Trial Questions } & \multicolumn{2}{c|}{ Status Trial Questions } \\
\hline $\begin{array}{l}\text { Left Agent expresses warmth } \\
\text { towards the Right Agent }\end{array}$ & $\begin{array}{l}\text { Right Agent expresses warmth } \\
\text { towards the Left Agent }\end{array}$ & $\begin{array}{l}\text { Left Agent tries to control his } \\
\text { interaction with Right Agent }\end{array}$ & $\begin{array}{l}\text { Right Agent tries to control his } \\
\text { interaction with Left Agent }\end{array}$ \\
\hline $\begin{array}{l}\text { Left Agent seems detached } \\
\text { from his interaction with Right } \\
\text { Agent }\end{array}$ & $\begin{array}{l}\text { Right Agent seems detached } \\
\text { from his interaction with Left } \\
\text { Agent }\end{array}$ & $\begin{array}{l}\text { Left Agent shows insecurity } \\
\text { in his interaction with Right } \\
\text { Agent }\end{array}$ & $\begin{array}{l}\text { Right Agent shows insecurity } \\
\text { in his interaction with Left } \\
\text { Agent }\end{array}$ \\
\hline $\begin{array}{l}\text { Left Agent tries to be likable } \\
\text { when interacting with Right }\end{array}$ & $\begin{array}{l}\text { Right Agent tries to be lik- } \\
\text { able when interacting with Left } \\
\text { Agent }\end{array}$ & $\begin{array}{l}\text { Left Agent is dominating the } \\
\text { interaction with Right Agent }\end{array}$ & $\begin{array}{l}\text { Right Agent is dominating the } \\
\text { interaction with Left Agent }\end{array}$ \\
\hline $\begin{array}{l}\text { Left Agent expresses hostility } \\
\text { towards Right Agent }\end{array}$ & $\begin{array}{l}\text { Right Agent expresses hostility } \\
\text { towards Left Agent }\end{array}$ & $\begin{array}{l}\text { Left Agent looks for reas- } \\
\text { surance while interacting with } \\
\text { Right Agent }\end{array}$ & $\begin{array}{l}\text { Right Agent looks for reas- } \\
\text { surance while interacting with } \\
\text { Left Agent }\end{array}$ \\
\hline
\end{tabular}

Table 1: On the left, the questionnaire used after each scene in the Affiliation trial and on the right, the questionnaire used in the Status trial.

$(M=.42, S E=.04)$. Consequently, H1.s and H2.s are supported by our experiment. An additional MANOVA with the group arrangement and the gender of the participant as between-subject factors revealed no significant differences (all $p>.07$ ).

\subsubsection{Affiliation trial.}

Our procedure is the same as in the Status Trial. Using a MANOVA $2 \times 2$, we first identified a main effect from the expressed Affiliation of Left Agent (WilksLambda $=0.44, F(2,10)=6.3$, $p<0.05$ ) but not from the expressed Affiliation of Right Agent (WilksLambda $=0.62, F(2,10)=3, p>0.05$ ). Again, no effect of the interaction of the variables could be identified.

Then, the conducted ANOVAs revealed that the expressed Affiliation of Left Agent had a significant effect on its measured Affiliation $(F(1,11)=13.2, p<.005)$, being higher when expressing a Friendly attitude $(M=.6, S E=.05)$ as opposed to an Hostile one $(M=.44, S E=.04)$.

The tests also revealed a significant effect of the expressed Affiliation of Right Agent on its measured Affiliation $(F(1,11)=6.2$, $p<.05)$ as its value was higher when the expressed attitude was a Friendly one ( $M=.57, S E=.05)$ as opposed to an Hostile one ( $M=.41, S E=.04)$. These results support H1.a and H2.a. An additional MANOVA with the group arrangement and the gender of the participant as between-subject factors also revealed no significant differences (all $p>.25$ ).

\section{Conclusion and Future Work}

We presented an evaluation study to investigate whether the attitudinal behaviors of a group of agents could be recognized by a user in a shared IVE. The agent model was adapted to the IVE in order to be able to produce the behaviors in real-time and to render the scene while taking into account the physical position of the user within it.

Results confirm that participants were able to recognize the attitudes expressed by the virtual agents in the group through a variety of nonverbal behaviors (c.f. Section 4). The Status level (respectively the Affiliation level) was rated significantly higher when the agents were expressing a Dominant (respectively Friendly) attitude rather than a Submissive (respectively Hostile) attitude. These results mirror those of our previous experiment where participants were looking at videos from generated scenes on a regular screen. These results allow us to assume that the attitudes, expressed by the behaviors produced by our model, could be identified when the user is observing the group from within the virtual environment.
Moreover, no statistical interaction between the variables has been found, which means that the attitude expressed by an agent might be correctly recognized independently of the attitude shown by the other agents.

There are some limitations that we aim to address in the future. First, our model focus mainly on producing behaviors to fulfill the role of the speaker. The turn-taking module computes the behaviors of the agent when it wants to take the turn, when it has it or when it is ending (or losing it). When the agent is a listener, only the gaze and body orientations are impacted. In order to address this limit, we aim to incorporate backchannels and to model explicitly listener's behavior. It could be interesting then to impact also the agent's prosody with the expression of attitudes, in both speaker and listener roles.

Secondly, in our scenario the user is merely an observer. Even though, in this immersive system, the agents share the virtual environment as the user and they place themselves to include him/her in their group, they do not involve the user in the conversation (in terms of turn-taking), or they do not take into account his/her behaviors while triggering their turn-taking behaviors. An interesting development could be the integration of a multi-party dialog manager that takes into account the behaviors of the users as well, such as in [Yumak et al. 2014].

\section{Acknowledgments}

This work was supported by national funds through Fundação para a Ciência e a Tecnologia (FCT) with reference UID/CEC/50021/2013, Télécom-ParisTech school (France) and the European Center for Virtual Reality of Brest (France).

\section{References}

Amatriain, X., Kuchera-Morin, J., Hollerer, T., AND POPE, S. T. 2009. The allosphere: Immersive multimedia for scientific discovery and artistic exploration. IEEE MultiMedia $16,2,0064-75$.

ARgYle, M. 1988. Bodily Communication. University paperbacks. Methuen.

Bailenson, J. N., Blascovich, J., Beall, A. C., AND LOOMIS, J. M. 2001. Equilibrium theory revisited: Mutual gaze and personal space in virtual environments. Presence 10,6, 583-598. 
Bombari, D., Mast, M. S., Canadas, E., And Bachmann, M. 2015. Studying social interactions through immersive virtual environment technology: virtues, pitfalls, and future challenges. Frontiers in psychology 6 .

BRADLEY, J. V. 1958. Complete counterbalancing of immediate sequential effects in a latin square design. Journal of the American Statistical Association 53, 282, 525-528.

Cruz-Neira, C., Sandin, D. J., And DeFanti, T. A. 1993. Surround-screen projection-based virtual reality: the design and implementation of the cave. In Proceedings of the 20th annual conference on Computer graphics and interactive techniques, ACM, 135-142.

Cummings, J. J., And Bailenson, J. N. 2015. How immersive is enough? a meta-analysis of the effect of immersive technology on user presence. Media Psychology, 1-38.

Diemer, J., Alpers, G. W., Peperkorn, H. M., Shiban, Y., AND MÜHLbERGER, A. 2015. The impact of perception and presence on emotional reactions: a review of research in virtual reality. Frontiers in psychology 6.

Fox, J., ArenA, D., And BAilenson, J. 2009. Virtual reality: A survival guide for the social scientist. Journal of Media Psychology 21, 3, 95-113.

Funder, D., Furr, R., AND Colvin, C. 2000. The riverside behavioral q-sort : a tool for the description of social behavior. Journal of Personality 68, 3, 451-489.

Goffman, E. 1981. Forms of talk. University of Pennsylvania Press.

Hall, E. T. 1969. The hidden dimension, vol. 1990. Anchor Books New York.

Hartanto, D., Brinkman, W.-P., Kampmann, I. L., MoRina, N., Emmelkamp, P. G. M., And NeerincX, M. A. 2015. Design and implementation of home-based virtual reality exposure therapy system with a virtual ecoach. In 15th International Conference on Intelligent Virtual Agents, Brinkman et al., Ed., vol. 9238 of LNCS, 287-291.

Heydarian, A., Carneiro, J. P., Gerber, D., BecerikGerber, B., Hayes, T., AND WoOd, W. 2014. Immersive virtual environments: experiments on impacting design and human building interaction. In Rethinking Comprehensive Design: Speculative Counterculture, Proceedings of the 19th International Conference on Computer-Aided Architectural Design Research in Asia (CAADRIA), 729-738.

Kendon, A. 1990. Conducting interaction: Patterns of behavior in focused encounters, vol. 7. CUP Archive.

Limniou, M., Roberts, D., And Papadopoulos, N. 2008. Full immersive virtual environment cave tm in chemistry education. Computers \& Education 51, 2, 584-593.

Lugrin, J.-L., Cavazza, M., Charles, F., Le Renard, M., FREEMAN, J., AND LESSITER, J. 2013. Immersive fps games: User experience and performance. In Proceedings of the 2013 ACM International Workshop on Immersive Media Experiences, ACM, New York, NY, USA, ImmersiveMe '13, 7-12.

McMahan, R. P., Bowman, D. A., Zielinski, D. J., And BRADY, R. B. 2012. Evaluating display fidelity and interaction fidelity in a virtual reality game. IEEE Transactions on Visualization and Computer Graphics 18, 4, 626-633.
Patel, K., Bailenson, J., HaCk-Jung, S., Diankov, R., And BAJCSY, R. 2006. The effects of fully immersive virtual reality on the learning of physical tasks. In PRESENCE 2006: The 9th Annual International Workshop on Presence.

Pecune, F., Cafaro, A., Chollet, M., Philippe, P., And Pelachaud, C. 2014. Suggestions for extending saiba with the vib platform. In Proceedings of the Workshop on Architectures and Standards for Intelligent Virtual Agents at IVA 2014.

Pedica, C., Vilhualmsson, H. H., And Larusdottir, M. 2010. Avatars in conversation: the importance of simulating territorial behavior. In Intelligent Virtual Agents, Springer, 336342.

Philipp, M. C., Storrs, K. R., And Vanman, E. J. 2012. Sociality of facial expressions in immersive virtual environments: A facial emg study. Biological psychology 91, 1, 17-21.

Ravenet, B., Ochs, M., And Pelachaud, C. 2013. From a user-created corpus of virtual agents non-verbal behavior to a computational model of interpersonal attitudes. In Intelligent Virtual Agents, Springer, 263-274.

Ravenet, B., Cafaro, A., Biancardi, B., Ochs, M., And Pelachaud, C. 2015. Conversational behavior reflecting interpersonal attitudes in small group interactions. In 15th International Conference on Intelligent Virtual Agents, vol. 9238 of LNCS, 375-388.

SADLER, P., AND WoOdY, E. 2010. Interpersonal complementarity. Handbook of interpersonal psychology : Theory, research, assessment, and therapeutic interventions, 123-127.

SCHERER, K. R. 2005. What are emotions? and how can they be measured? Social science information 44, 4, 695-729.

Slater, M., Steed, A., And Usoh, M. 2013. Being There Together: Experiments on Presence in Virtual Environments (1990s). Technical Report, Department of Computer Science, University College London, UK.

Thórisson, K. R., Gislason, O., Jonsdottir, G. R., And THORISSON, H. T. 2010. A multiparty multimodal architecture for realtime turntaking. In Intelligent Virtual Agents, Springer, $350-356$.

Yumak, Z., Ren, J., Thalmann, N. M., And Yuan, J. 2014. Modelling multi-party interactions among virtual characters, robots, and humans. Presence: Teleoperators and Virtual Environments 23, 2, 172-190. 